\title{
INDIVIDUAL TREE DETECTION AND DETERMINATION OF TREE PARAMETERS USING UAV-BASED LIDAR DATA
}

\author{
F. Bayat ${ }^{1}$, H. Arefi ${ }^{1, *}$, F. Alidoost ${ }^{1}$ \\ ${ }^{1}$ School of Surveying and Geospatial Engineering, College of Engineering, University of Tehran, Iran - \\ (bayatfariba, hossein.arefi, falidoost)@ut.ac.ir
}

KEY WORDS: Forest Inventory, LiDAR, Tree Detection, UAV, Point Classification

\begin{abstract}
:
Forest inventory provides comprehensive information about the geometric and biometric state of forests as well as vegetated areas. In this study, a point-based 3D method is presented for tree detection as well as measuring of structural properties of forests such as the tree height, tree position and canopy area using high resolution point cloud which is provided by an Unmanned Aerial Vehicle (UAV)-based LiDAR sensor. The proposed method is based on the density of point cloud and 2D and 3D distance measurements. It includes three main steps as pre-processing, tree detection, and extraction of tree structural attributes. After generating a canopy height model, an image is created based on the density of point cloud. Next, points are classified based on 2D and 3D distance measurements, sequentially, from the highest to the lowest. According to the results, the rate of tree detection is about $95 \%$ and the main structural parameters of a tree such as the position, height, area and length of the canopy are estimated with the accuracy of $1.97 \mathrm{~m}, 0.36 \mathrm{~m}, 12.78 \mathrm{~m} 2$ and $0.79 \mathrm{~m}$, respectively.
\end{abstract}

\section{INTRODUCTION}

Nowadays, forests and natural ecosystems have a vital role in sustainable development for countries. In order to manage and protect these invaluable resources, it is essential to measure the specific characteristics of forests such as the type of the forest soil, type and age of plants as well as position and status of individual trees. Forest inventory is to extract information about forest growth, structural information on forest mass, the volume of trees, and so on. The structural information of the forest mass indicates the spatial arrangement of the forest components and also describes some of the features, such as horizontal distribution, vertical distribution, and abundance of plant species (Wulder, et al., 2008). However, measuring of structural information of forests using terrestrial and manual approaches is not affordable. On the other hand, due to significant advances in remotely sensed data acquisition techniques, these technologies have been widely applied to characterize the 3D structure of trees and extract the forest information (Wulder, et al., 2008; Li, et al., 2012). One of the remote sensing technologies, which is increasingly used in 3D forest inventory application, is LiDAR technology that can provide a precise estimation of both vegetation and terrain height ( $\mathrm{Li}$, et al., 2012). However, in color-based imaging methods, more detailed spectral information can be extracted from top layers of tree crown, but two-dimensional image processing and extraction of elevation information require further computational time and power, and even with a high interpolation between the obtained images, and as a result, with higher density of resulting point clouds, these methods cannot provide a full definition of the vertical profile of the plants and reach the same level of information on the vertical classification of vegetation and terrain layers in the Lidar data.
The parameters of the forest structure such as tree diameter at breast height, mean height, dominant height, crown density, crown volume, crown area, and biomass, as well as parameters of the trunk of trees such as length, density, radial stem diameter, and wood thickness can be measured using LiDAR data. The terrestrial laser scanners are capable to collect highdetailed information from the lower and middle layers of plants such as stem diameter. One of the disadvantages of a terrestrial sensor is that it should be positioned on a tripod and remain fixed during data gathering. Therefore, they are mostly used to collect data from a small area. On the other hand, aerial laser scanners (e.g. LiDAR) are utilized to collect data from a large area, covered by trees, with a density of less than 1 point per $\mathrm{m} 2$. However, the aerial technology suffers from high operating cost. Recently, the UAVs are used as a new platform for carrying the Lidar sensors and acquiring data from forest areas. The LiDAR UAVs can be utilized in applications such as measuring the health parameters of forest and tree crown situations. The advantages of LiDAR UAVs compared to other platforms are the lower operating costs, the higher flexibility of movement in the forests, multiple mapping, and easy use and easy transportation. Using LiDAR UAVs, there is the possibility of flying at low elevation, and consequently, the density of points can be increased for example up to $150 \mathrm{dot} / \mathrm{m} 2$ in the forest areas. In addition, the high data density is helpful to reduce the errors and increase the information from the lower layers of the plants.

According to the literature studies, there are two groups of methods in forest inventory as area-based approaches (ABA) (Hadaś, 2015; Kathuria, et al., 2016 and Individual Tree Detection (ITD) approaches (Hyyppä, et al., 1999; Brandtberg, et al., 2003; Teixeira de Oliveira, et al., 2012). The aim of this research is to propose an optimal method for detection of individual trees and extraction of statistical tree information

\footnotetext{
* Corresponding author
} 
such as height, position, and width of the tree crown. In this study, the data is collected by a UAV-based Lidar sensor from a densely covered forest area. Since the proposed method is based on $2 \mathrm{D}$ and $3 \mathrm{D}$ distances between points. Therefore, the final results don't suffer from the interpolation errors.

\section{PROPOSED METHOD}

The proposed method contains three steps as data preprocessing, individual tree detection and tree's parameters calculation. Each step is described as follows.

\subsection{Data pre-processing}

In the pre-processing step, the blunder points are first detected and removed from the data. For blunder detection, a neighboring window with a pre-defined size is applied to each point and the points with elevation values larger than the average height of the neighborhood are eliminated. Next, the remained points are classified into terrain and non-terrain points based on thresholding on height values and a normalized Digital Surface Model (nDSM) is generated using tree points.

\subsection{Individual tree detection}

As shown in Figure 1, the proposed method for individual tree detection is based on $2 \mathrm{D}$ and $3 \mathrm{D}$ distances between LiDAR points, inspired by ( $\mathrm{Li}$, et al., 2012; $\mathrm{Lu}$ et al., 2014) and includes three sub-steps. In this method, the points are first extracted along the trunk of trees and then the other points are added to these points using the distance-based rules.

In the first step of tree detection, the points of each tree are sorted based on elevation values and in the descending mode. Then, an image is generated in a perpendicular direction of the elevation values. Each pixel of that image is initialized based on the number of points in a pixel. Since the density of points along the trunk is higher than other locations, the pixels with larger values belong to the trunk of tree. Next, the point clustering is performed based on the distances of points, iteratively. If the number of iteration (which is equal to the number of trees) is $\boldsymbol{i}$, the set of points is $\boldsymbol{W}$, the set of identified tree points is $\boldsymbol{T}$, and the set of unidentified tree points is $\boldsymbol{U}$, in each iteration, points are classified into two classes of $\boldsymbol{T}$ and $\boldsymbol{U}$ based on a to-down classification technique. For this, the identified trunk points are first transferred into the $\boldsymbol{T}$, and one point, $\boldsymbol{a} \in \boldsymbol{U}$, is selected so that the distance between $\boldsymbol{a}$ and the highest point in the $\boldsymbol{T}$ is equal to $100 \mathrm{~m}$. The $\boldsymbol{d}_{\boldsymbol{l}}$ and $\boldsymbol{d}_{2}$ distances are then calculated which are the minimum 2D distances of $\boldsymbol{a}$ from $\boldsymbol{T}$ and $\boldsymbol{U}$, respectively. Finally, if $\boldsymbol{a}$ is a highest point in a neighborhood with the radius of $\boldsymbol{R}$, the point of $\boldsymbol{a}$ is classified as follow. Where $\boldsymbol{d}_{\boldsymbol{u}}$ is a predefined threshold.

$$
\begin{aligned}
& \text { if } d_{1}>d_{u} \quad a \in U_{i} \\
& \text { if } d_{1}<=d_{u} \text { and } d_{1}<=d_{2} \quad a \in T_{i} \\
& \text { if } d_{1}<=d_{u} \text { and } d_{1}>d_{2} \quad a \in U_{i}
\end{aligned}
$$

Otherwise, the point $\boldsymbol{a}$ is classified as follows.

$$
\begin{aligned}
& \text { if } \quad d_{1}<=d_{2} \quad a \in T_{i} \\
& \text { if } \quad d_{1}>d_{2} \quad a \in U_{i}
\end{aligned}
$$

In each iteration, only one point is identified and removed from the $\boldsymbol{U}$. At the end of iterations, some points are remained in the $\boldsymbol{U}$ as undetected points which are classified in the second step. In the second step of tree detection, the 3D distances between $\boldsymbol{a} \in \boldsymbol{U}$, and all points in $\boldsymbol{T}$ are calculated and a tree, $\boldsymbol{C} \in \boldsymbol{T}$, with the minimum distance from $\boldsymbol{a}$ is selected. Then, the 2D distances between $\boldsymbol{a}$ and all points of $\boldsymbol{C}$ are calculated. If the minimum distance, $\boldsymbol{d}$, is lower than a pre-defined threshold, the $\boldsymbol{a}$ is classified as a point of $\boldsymbol{C}$. Otherwise, $\boldsymbol{a}$ is remained in the $\boldsymbol{U}$ as an undetected point.

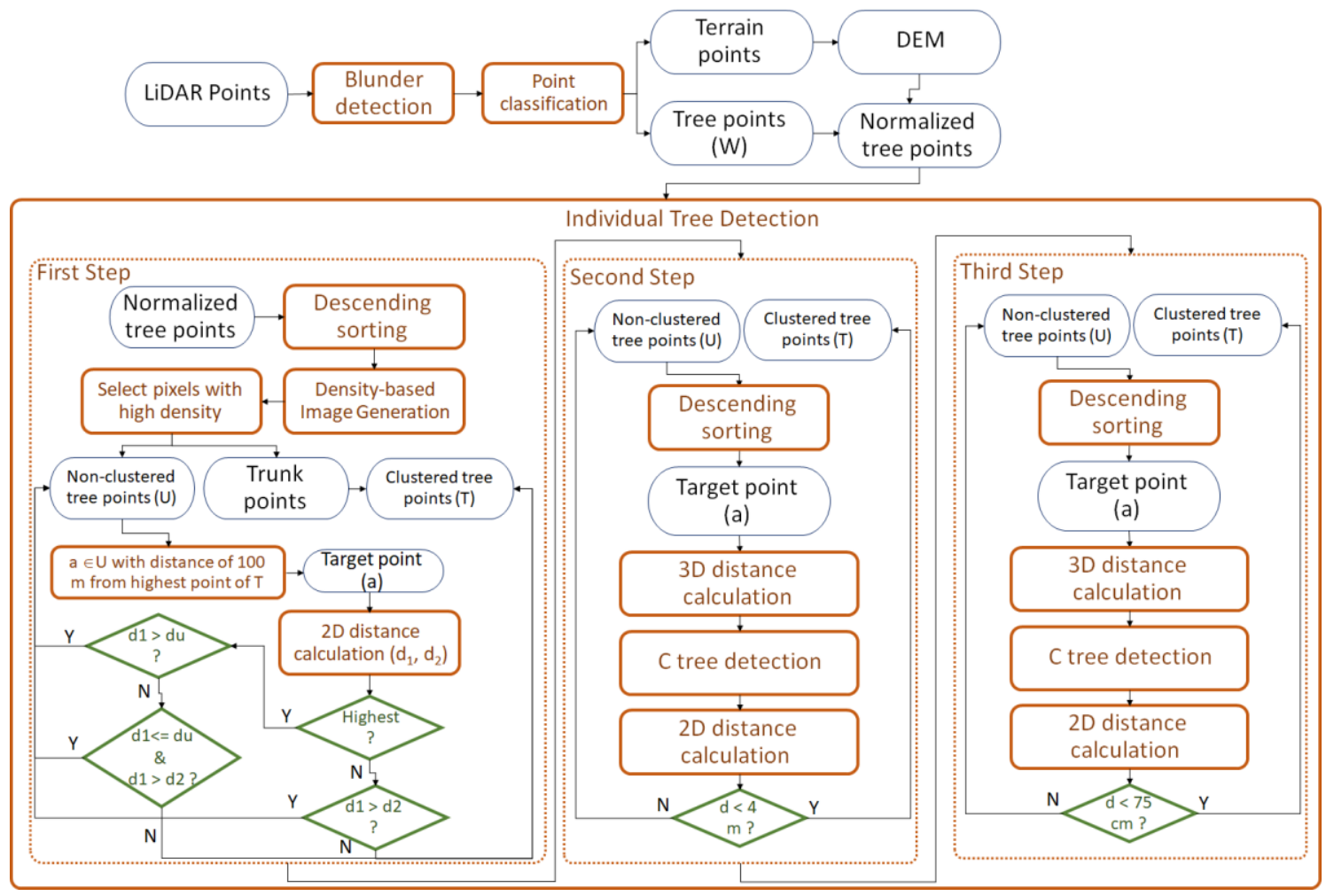

Figure 1. The proposed method for ITD 
Finally, in the third step of tree detection, the 3D distances between $\boldsymbol{a} \in \boldsymbol{U}$, and all points in $\boldsymbol{T}$ are only calculated. If the 3D distance between a and a tree in $\boldsymbol{T}$ is lower than a pre-defined threshold, the $\boldsymbol{a}$ is classified as a point of that tree. Otherwise, $\boldsymbol{a}$ is remained in the $\boldsymbol{U}$ as an undetected point.

\subsection{Tree's parameters calculation}

The tree's parameters such as the position, height, area as well as the width of the tree crown can be measured based on classified points for individual trees. For each tree, the height and the location of the point with the maximum elevation is used as the height and the location of that tree. In addition, the area of the crown is equal to the area of a convex hull of points and the width of the crown is the average distance between the centre of tree and other points (Figure 2).

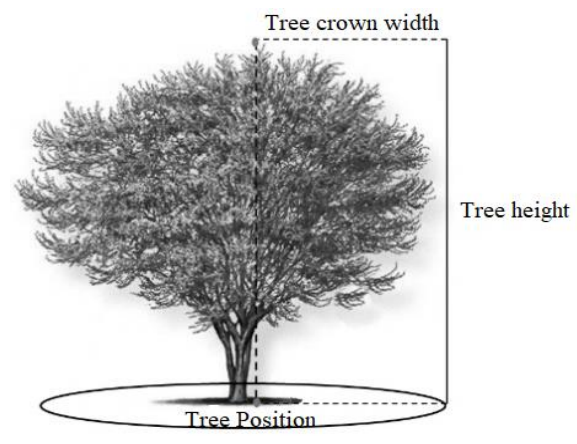

Figure 2. The tree's parameters definition

\section{EXPERIMENTS AND RESULTS}

In this study, a dataset over a forest area of $6500 \mathrm{~m}^{2}$ including different trees is selected to evaluate the proposed method. The dataset is collected by a laser scanner mounted on a UAV so that the horizontal and vertical accuracies as well as the density of points are about $0.01-0.02 \mathrm{~m}, 0.02 \mathrm{~m}$ and $12 \mathrm{dot} / \mathrm{m}^{2}$, respectively.

In the pre-processing step, the points in a neighborhood of $5 \mathrm{~m}$ are compared and points with the height larger than $25 \mathrm{~m}$ are classified as the blunders. Moreover, the points with the minimum height in each neighborhood are considered as the terrain points and an nDSM is generated with the spatial resolution of $0.3 \mathrm{~m}$. In the tree detection, the predefined thresholds are selected as $0.75 \mathrm{~m}, 4 \mathrm{~m}$, and $0.75 \mathrm{~m}$ for each steps, respectively. The results of the tree detection are shown in Figure 3.

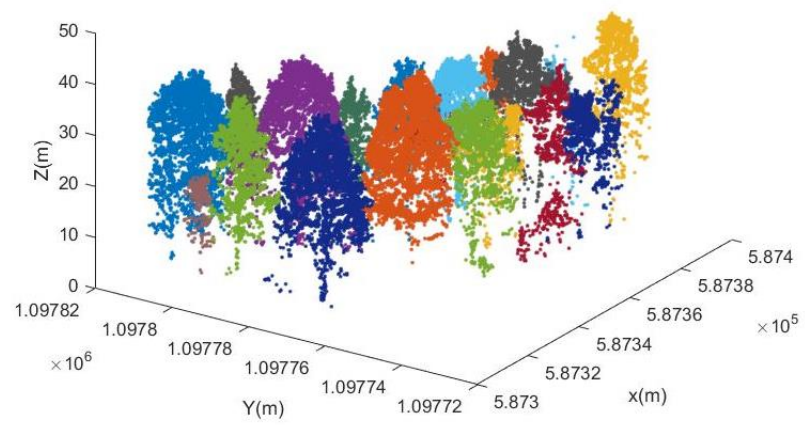

(a)

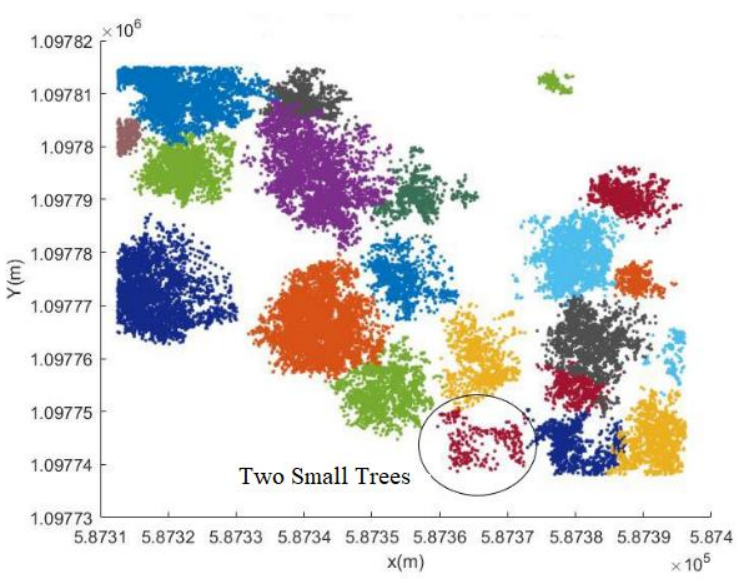

(b)

Figure 3. A 3D (a) and 2D (b) mode of the final results for tree detection

In order to evaluate the accuracy of the individual tree detection step as well as the calculated parameters, a ground truth is generated manually and visually. For tree detection, three metrics as the detection rate, $\boldsymbol{r}$, the detection accuracy, $\boldsymbol{p}$, and the overall accuracy, $\boldsymbol{F}$, are calculated by given Eq. 3 .

$$
\begin{aligned}
& r=\frac{T P}{T P+F N} \\
& p=\frac{T P}{T P+F P} \\
& F=2 * \frac{r * p}{r+p}
\end{aligned}
$$

Where $\boldsymbol{T P}$ is the number of trees that are correctly identified, $\boldsymbol{F P}$ is the number of trees which are not correctly identified and the $\boldsymbol{F N}$ is the number of the unclassified points. Since the total number of tress is 23 and the number of detected trees is 22 in the case study, the values for $\boldsymbol{r}, \boldsymbol{p}$ and $\boldsymbol{F}$ are same and it is $95 \%$. Moreover, three metrics as the root mean square error $(\boldsymbol{R M S E})$, Bias, and linear correlation (coefficient of explanation) $\left(\boldsymbol{r}^{2}\right)$ are calculated as follows to evaluate the accuracy of tree's parameters.

$$
\begin{aligned}
& R M S E=\sqrt{\frac{\sum_{i=1}^{n}\left(x_{i r}-x_{i f}\right)}{n}} \\
& \text { Bias }=\frac{\sum_{i=1}^{n}\left(x_{i r}-x_{i f}\right)}{n} \\
& r^{2}=\operatorname{cor}\left(x_{i r}, x_{i f}\right)^{2}
\end{aligned}
$$

Where $\boldsymbol{n}$ is the number of observations, $\boldsymbol{x}_{i r}$ is the real value and the $\boldsymbol{x}_{i f}$ is the calculated values for each parameter. In Figure 4, the measurement method for height of trees and the area of tree crown are shown. The quantitative results are also presented in Table 1 .

\begin{tabular}{|l|c|c|c|}
\hline Tree's Parameters & \multicolumn{3}{|c|}{ Metrics } \\
\hline & RMSE & Bias & $\mathrm{r}^{2}$ \\
Position & $1.97 \mathrm{~m}$ & $0.57 \mathrm{~m}$ & 0.99 \\
Height & $0.36 \mathrm{~m}$ & $0.12 \mathrm{~m}$ & 0.99 \\
Crown width & $0.79 \mathrm{~m}$ & $0.61 \mathrm{~m}$ & 0.91 \\
Crown area & $12.7 \mathrm{~m}^{2}$ & $8.12 \mathrm{~m}^{2}$ & 0.93 \\
\hline
\end{tabular}

Table 1 . The accuracy of tree's parameters 


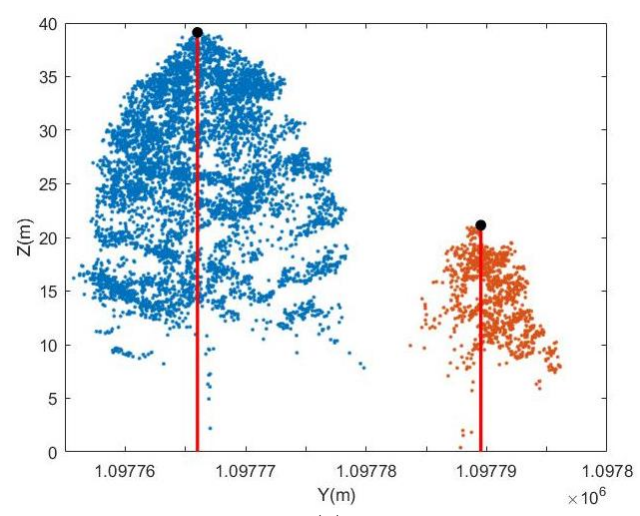

(a)

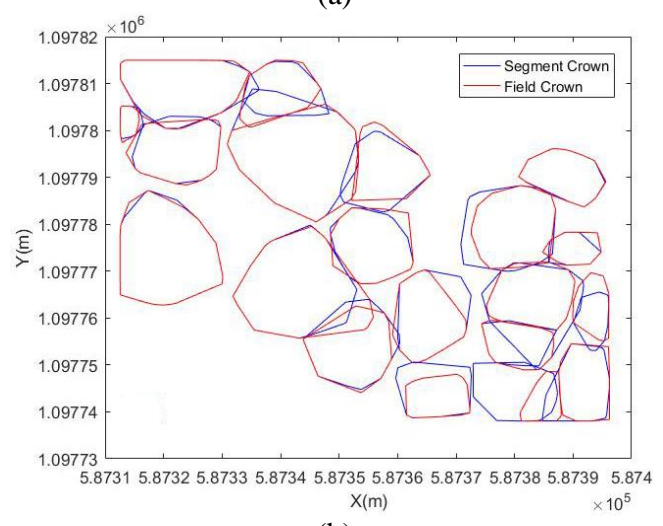

(b)

Figure 4. The measurements of tree heights (a) and crown's areas (b)

\section{CONCLUSIONS}

In this study, a point-based method is presented for individual tree detection using LiDAR data. The proposed method is only based on 2D and 3D distances between points as well as the point density in different locations of a tree. A set of geometric rules are applied to distances to classify tree points. According to the detected points for each tree, the vital parameters such as the position, height, and the width of the tree crown can be measured with the RMSE of less than $2 \mathrm{~m}$.

\section{REFERENCES}

Wulder M.A., Bater C.W., Coops N. C., Hilker T. and White J.C., 2008. The role of LiDAR in sustainable forest management. The Forestry Chronicle, 84(6): 807-826.

Li W., Guo Q., Jakubowski M.K., and Kelly M., 2012. A New method for segmenting individual trees from the LiDAR point cloud. Photogrammetric Engineering \& Remote Sensing 78(1): 75-84.

Hadaś E., 2015. An overview of methods for tree geometric parameter estimation from ALS data in the context of their application for agricultural trees, Acta Sci. Pol., 14 (3-4): 5-28.

Kathuria A., Turner R., Stone Ch., Duque-Lazo J. and West R. 2016. Development of an automated individual tree detection model using point cloud LiDAR data for accurate tree counts in a pinus radiata plantation, Australian Forestry, 79(2):126-136.
Teixeira de Oliveira L., Marcelo Tavares de Carvalho L., Zélia Ferreira M., Chaves de Andrade Oliveira T. and Weimar Acerbi Junior F., 2012. Application of LiDAR to forest inventory for tree count 17 in stands of eucalyptus sp. CERNE, 18(2):175184.

Brandtberg T., Warnera T.A., Landenbergerb R.E. and McGraw J.B., 2003. Detection and analysis of individual leaf-off tree crowns in small footprint, high sampling density LiDAR data from the eastern deciduous forest in North America. Remote Sens. Environ., 85(3): 290-303.

Hyyppä, J. and Inkinen, M., 1999. Detecting and estimating attributes for single trees using laser scanner. Photogramm. J. Finl. 1999, 16, 27-42.

Lu X., Guo Q., Li W. and Flanagan J., 2014. A bottom-up approach to segment individual deciduous trees using leaf-off LiDAR point cloud data. ISPRS Journal of Photogrammetry and Remote Sensing, 94: 1-12. 